\title{
Variations in breathing patterns increase low frequency contents in HRV spectra
}

\author{
M A García-González†, C Vázquez-Seisdedos $\dagger+$ and R Pallàs-Areny $\dagger$ \\ $\dagger$ Instrumentation and Bioengineering Division (DIB), Department of Electronic Engineering \\ (DEE), Universitat Politècnica de Catalunya (UPC), C/Jordi Girona 1-3, Campus Nord Edifici \\ C-4, 08034 Barcelona, Spain \\ $\ddagger$ Department of Telecommunication, Universidad de Oriente, 90900 Santiago de Cuba, Cuba \\ E-mail: magarcia@eel.upc.es
}

Received 20 April 2000

\begin{abstract}
This paper shows that variations in breathing patterns broaden heart rate variability (HRV) spectral bands and increase the power amplitude of low-frequency bands. Because of these influences, spectral markers for HRV signals, such as the quotient between spectral power at different frequency bands, should be compared only under controlled breathing conditions or after considering the effect of variations in breathing patterns.
\end{abstract}

Keywords: heart rate variability, spectral analysis, respiratory sinus arrhythmia, controlled respiration

\section{Introduction}

Heart rate variability (HRV) analysis has become a useful tool to study the relationship between the autonomic nervous system (ANS) and the cardiovascular system (CS). HRV is usually studied from the RR time series, either by time-domain methods (Kleiger et al 1993), nonlinear dynamics (Wada et al 1993), or spectral analysis (Pagani et al 1986). HRV analysis has been applied, for example, to study the risk of cardiac electrical instability after myocardial infarction (Bigger et al 1991), to diagnose diabetic neuropathy (Pagani et al 1988), to assess re-innervation after cardiac transplantation (Sands et al 1989) and to evaluate exercise training (Arai et al 1989).

Spectral analysis describes the frequency distribution of signal power. Analysis of rhythmic events in the RR time series permits us to infer the state of biological oscillators that modulate the CS, the sympathetic and vagal efferent activity, humoral factors and the sinus node (Task Force 1996). Usually, the power spectrum of the RR time series is divided into three bands: the very-low-frequency (VLF) band, the low-frequency (LF) band, and the high-frequency (HF) band. Vagal activity is the major contributor to the HF component mostly because of the respiratory sinus arrhythmia (Pomeranz et al 1985). The LF band is assumed to reflect sympathetic modulations or both sympathetic and vagal activity. In HRV spectral analysis, the biological oscillators that modulate the CS are commonly considered to remain invariable. Nevertheless, the amplitude and frequency of that modulation is seldom stationary in practice. 
This work investigates the effects of breathing-frequency variations in the spectral quantification of HRV signals. Respiratory activity causes a modulation in RR time series at the breathing frequency, which is termed respiratory sinus arrhythmia. The amplitude of this modulation depends on vagal tone (Kollai and Mizsei 1990) and on the breathing frequency itself (Womack 1971) (high breathing frequencies yield smaller amplitude oscillations in the RR spectrum than low breathing frequencies). Hence, changes in breathing frequency should be reflected in the RR time series as a modulation in both amplitude and frequency, and result in a modified spectrum of the RR time series as compared to that obtained during periodic breathing. Consequently, we can expect that variations in breathing patterns change spectral markers in both the HF and LF bands of RR time series spectra. These changes exclusively due to the breathing pattern (i.e. the cause) should not be mistaken for abnormal changes in HRV (i.e. changes in the cause-to-effect relationship).

This paper shows that variations of the breathing frequency increase the LF/HF index. First, the theoretical spectrum of FM and low-pass filtered signals is presented in order to substantiate the working hypothesis. Next, spectral estimation methods of actual time series are briefly discussed in order to justify the spectral analysis method used here. Finally, simulation and experimental results are presented and discussed in order to draw some conclusions.

\section{Theoretical spectrum of FM and low-pass filtered signals}

The spectrum of a sine wave whose frequency is $f_{\mathrm{c}}$ and variance $\sigma_{\mathrm{c}}^{2}$,

$$
x_{\mathrm{c}}(t)=\frac{\sigma_{\mathrm{c}}}{\sqrt{2}} \cos \left(2 \pi f_{\mathrm{c}} t\right)
$$

is a spectral peak located at $f_{\mathrm{c}}$. If $f_{\mathrm{c}}$ varies with time, the signal spectrum changes. Consider for example the frequency modulated (FM) signal

$$
x_{\mathrm{FM}}(t)=\frac{\sigma_{\mathrm{c}}}{\sqrt{2}} \cos \left(2 \pi f_{\mathrm{c}} t+2 \pi f_{\Delta} \int x_{\mathrm{m}}(\lambda) \mathrm{d} \lambda\right)
$$

where $f_{\Delta}$ is the frequency deviation and $x_{\mathrm{m}}(t)$ is the modulating signal with variance $\sigma_{\mathrm{m}}^{2}$ and probability distribution function (PDF) $p_{x_{\mathrm{m}}}(x)$. The quasi-static approximation (Peebles 1976) shows that the two-sided power spectrum of $x_{\mathrm{FM}}(t)$ when the frequency of variation is slow compared to $f_{\mathrm{c}}$ is

$$
S_{x_{\mathrm{FM}} x_{\mathrm{FM}}}(f)=\frac{\sigma_{\mathrm{c}}^{2} \sigma_{\mathrm{m}}^{2}}{2 f_{\Delta}}\left[p_{x_{\mathrm{m}}}\left(\frac{f-f_{\mathrm{c}}}{f_{\Delta}}\right)+p_{x_{\mathrm{m}}}\left(\frac{f+f_{\mathrm{c}}}{f_{\Delta}}\right)\right] .
$$

Therefore, frequency variation implies a broadening of the spectral peak, which depends on the PDF of the modulating signal.

Since there is a low-pass filtering action between breathing frequency and heart rate fluctuation (Womack 1971), we are interested in the spectrum of the output $y(t)$ of that filter, which is

$$
S_{y}(f)=\frac{\sigma_{\mathrm{c}}^{2} \sigma_{\mathrm{m}}^{2}}{2 f_{\Delta}}\left[p_{x_{\mathrm{m}}}\left(\frac{f-f_{\mathrm{c}}}{f_{\Delta}}\right)+p_{x_{\mathrm{m}}}\left(\frac{f+f_{\mathrm{c}}}{f_{\Delta}}\right)\right]\left|H_{\mathrm{LP}}(f)\right|^{2} .
$$

That is, the power spectrum of $y(t)$ is that of $x_{\mathrm{FM}}$ weighted by the filter transfer function. Hence, the low-frequency components of $x_{\mathrm{FM}}(t)$ will be enhanced as compared to the high-frequency components. 


\section{Spectral estimation of RR time series}

The spectral estimation of time series must consider the finite number of samples available and the sampling process itself. In an RR time series (tachogram), the information is provided by each QRS arrival, which results in non-uniform sampling, leading to spectral artifacts (Mateo and Laguna 2000). Resampling the tachogram with splines yields a uniformly sampled and interpolated RR time series, which avoids artifacts.

Spectral estimation methods can be either non-parametric, such as the periodogram and its modifications (Barlett, Welch and Blackman-Tukey periodograms), or parametric (AR, MA and ARMA modelling). The need for high-frequency resolution, the finite length of the time series, the smoothness of the spectra and its capability of identifying periodical components immersed in noise favoured the extended use of parametric estimation methods. Nevertheless, non-parametric methods are more accurate than parametric methods to quantify power distribution in frequency bands (Mateo and Laguna 2000), which is the basis for markers in HRV spectral analysis. Hence, we estimate the spectra from the periodogram (squared modulus of the FFT) of the tachogram resampled and interpolated by cubic splines.

Finally, frequency bands in HRV variability analysis must be delimited according to the intended application. Since the narrower the LF band, the smaller the LF/HF index, we normalize the LF/HF index to the signal bandwidth according to

$$
\mathrm{LF} / \mathrm{HF}_{\mathrm{n}}=\frac{S_{\mathrm{LF}}}{S_{\mathrm{HF}}} \frac{\mathrm{BW}_{\mathrm{HF}}}{\mathrm{BW}_{\mathrm{LF}}} .
$$

We are interested in variations in the $\mathrm{LF} / \mathrm{HF}_{\mathrm{n}}$ index because of variations in breathing frequency. Therefore, we will analyse RR time series during different breathing patterns that involve respiratory frequencies from $0.1 \mathrm{~Hz}$ to $0.2 \mathrm{~Hz}$. Consequently, we define the three following frequency bands (see figure 1):

$$
\begin{cases}f \leqslant 0.03 \mathrm{~Hz} & \text { VLF band } \\ 0.03 \mathrm{~Hz}<f \leqslant 0.09 \mathrm{~Hz} & \text { LF band } \\ 0.09 \mathrm{~Hz}<f<0.4 \mathrm{~Hz} & \text { HF band. }\end{cases}
$$

According to this definition, $\mathrm{BW}_{\mathrm{LF}}=0.06 \mathrm{~Hz}$ and $\mathrm{BW}_{\mathrm{HF}}=0.31 \mathrm{~Hz}$.

\section{Results from simulated tachograms}

The first step in assessing how variations in respiratory frequency affect the $\mathrm{LF} / \mathrm{HF}_{\mathrm{n}}$ index is to analyse simulated tachograms that include different beat-to-beat interval modulations. A 5 min fragment of a periodic RR signal (in milliseconds) with $f_{\mathrm{c}}=0.15 \mathrm{~Hz}$ is

$$
\operatorname{RR}_{\mathrm{p}}(n)=1000+100 \cos (2 \pi \times 0.15 n) \quad n \in[1, \ldots, 300]
$$

where $n$ is the heartbeat number. Hence, the shortest RR interval is $900 \mathrm{~ms}$, the longest is $1100 \mathrm{~ms}$ and the average is $1000 \mathrm{~ms}$. A 5 min fragment of an FM modulated RR signal (in milliseconds) with the same maximum, minimum and mean of $\mathrm{RR}_{\mathrm{p}}$ and with $f_{\mathrm{c}}=0.15 \mathrm{~Hz}$, $f_{\Delta}=0.05 \mathrm{~Hz}$ and $f_{\mathrm{m}}=0.01 \mathrm{~Hz}$ is

$$
\begin{gathered}
\operatorname{RR}_{\mathrm{FM}}(n)=1000+100 \cos \left[2 \pi \times 0.15 n+2 \pi \times 0.05 \sum_{i=1}^{n} \cos (2 \pi \times 0.01 \mathrm{i})\right] \\
n \in[1, \ldots, 300] .
\end{gathered}
$$

Both tachograms are resampled at $4 \mathrm{~Hz}$ prior to the spectral estimation. Figure 1 shows that the respective frequency spectra are quite different in spite of the average breathing frequency 


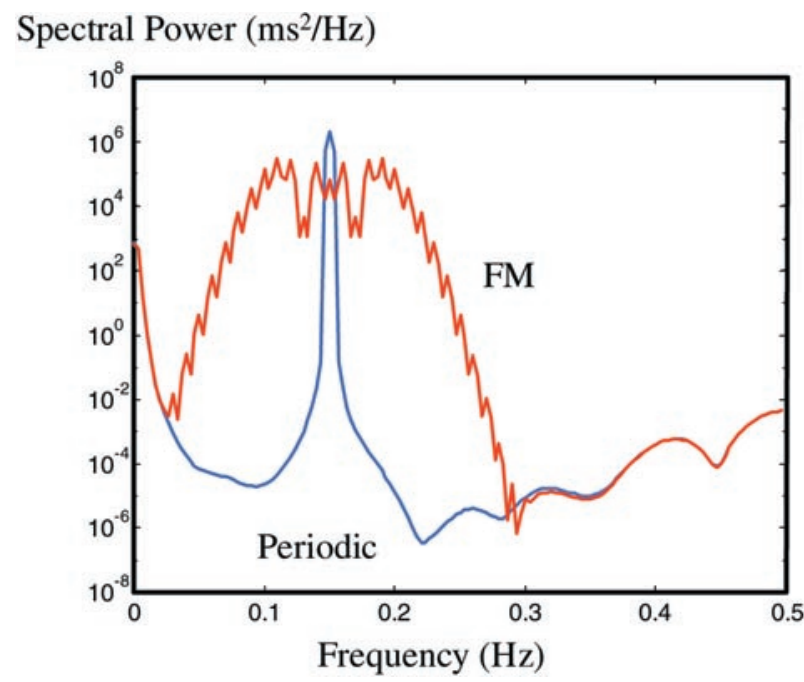

Figure 1. Spectra of simulated $R R_{F M}$ and $R R_{p}$.

Table 1. Normalized power-spectra ratio index $\left(\mathrm{LF} / \mathrm{HF}_{\mathrm{n}}\right)$ for the $\mathrm{RR}$ time series under periodic $\left(R_{\mathrm{p}}\right)$ and $\mathrm{FM}$ modulated $\left(\mathrm{RR}_{\mathrm{FM}}\right)$ breathing after low-pass filtering with different cut-off frequencies.

\begin{tabular}{lllllll}
\hline & $0.05 \mathrm{~Hz}$ & $0.07 \mathrm{~Hz}$ & $0.10 \mathrm{~Hz}$ & $0.13 \mathrm{~Hz}$ & $0.15 \mathrm{~Hz}$ & $0.20 \mathrm{~Hz}$ \\
\hline Periodic & $796 \times 10^{-6}$ & $6.9 \times 10^{-6}$ & $0.7 \times 10^{-6}$ & $5.80 \times 10^{-9}$ & $2.07 \times 10^{-9}$ & $3.62 \times 10^{-6}$ \\
FM & 0.68 & 0.48 & 0.23 & 0.12 & 0.09 & 0.05 \\
\hline
\end{tabular}

being the same $(0.15 \mathrm{~Hz})$. The $\mathrm{LF} / \mathrm{HF}_{\mathrm{n}}$ index is also very different: 0.03 for $\mathrm{RR}_{\mathrm{FM}}$ and $7 \times 10^{-9}$ for $\mathrm{RR}_{\mathrm{p}}$.

An improved simulation includes the low-pass filtering effect reported by Womack (1971). We have computed the $\mathrm{LF} / \mathrm{HF}_{\mathrm{n}}$ index for the outputs of several low-pass filters whose inputs were $\mathrm{RR}_{\mathrm{p}}$ and $\mathrm{RR}_{\mathrm{FM}}$. The filters used were Butterworth fourth-order and bidirectional, with cutoff frequencies $0.05 \mathrm{~Hz}, 0.07 \mathrm{~Hz}, 0.10 \mathrm{~Hz}, 0.13 \mathrm{~Hz}, 0.15 \mathrm{~Hz}$ and $0.20 \mathrm{~Hz}$. Table 1 shows the results. For the $\mathrm{RR}_{\mathrm{FM}}$ signal, the lower the cut-off frequency, the higher the $\mathrm{LF} / \mathrm{HF}_{\mathrm{n}}$ index. For the $\mathrm{RR}_{\mathrm{p}}$ signal, the cut-off frequency strongly affects the $\mathrm{LF} / \mathrm{HF}_{\mathrm{n}}$ index because of the small power in the $\mathrm{LF}$ band. In any case, the $\mathrm{LF} / \mathrm{HF}_{\mathrm{n}}$ index is always smaller for periodic $\mathrm{RR}$ signals than for FM RR signals.

Actual RR signals will be influenced by other heart rate variability factors in addition to the respiration. Therefore, in order to consider the spectral power in the LF band resulting only from the breathing pattern, we will high-pass filter the tachogram before performing spectral analysis. We have filtered the signals with a Butterworth fourth-order bidirectional high-pass filter with a cut-off frequency of $0.05 \mathrm{~Hz}$. The results show that this filter has a negligible effect on the $\mathrm{LF} / \mathrm{HF}_{\mathrm{n}}$ index for the simulated signals.

\section{Experimental results}

We have recorded RR time series and breathing signals in 20 healthy subjects ( $30.9 \pm 5.1$ years) in supine position. Measurements for each subject induced five stages with well defined conditions. Each stage lasted for 5 minutes and was followed by a 2 minute resting interval. 


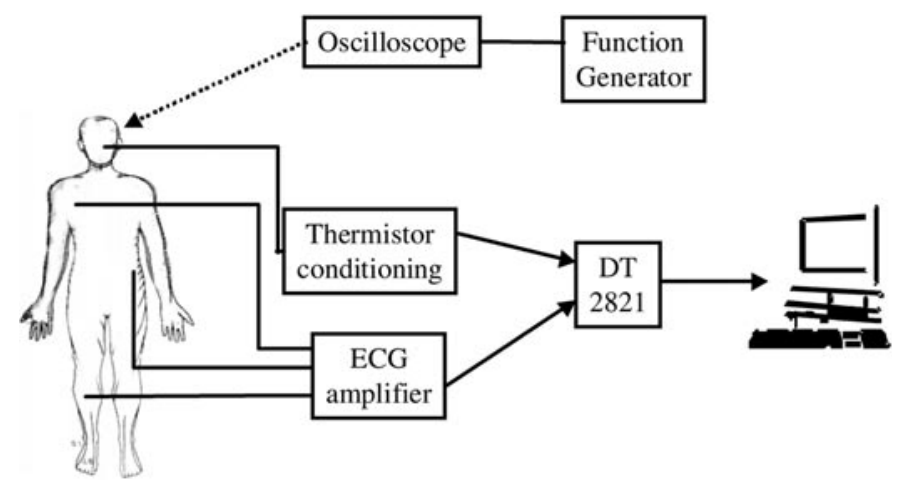

Figure 2. Measurement system.

Before starting, subjects were in supine position for 5 minutes. In the first stage, subjects breathed at will. In the second stage, subjects were asked to breathe periodically at $0.15 \mathrm{~Hz}$ by following a signal on an oscilloscope screen. In the third stage, subjects were asked to breathe according to an oscilloscope displaying a $0.15 \mathrm{~Hz}$ signal modulated in frequency by $0.01 \mathrm{~Hz}$ with a $0.05 \mathrm{~Hz}$ frequency deviation. In the fourth stage, subjects returned to periodical breathing at $0.15 \mathrm{~Hz}$, and during the fifth stage, subjects breathed at will again.

Figure 2 shows the measurement setup. The isolated ECG amplifier (bandwidth $0.5 \mathrm{~Hz}$ to $500 \mathrm{~Hz}$, gain 1000) recorded the standard II ECG lead. A nasal NTC thermistor yielded the breathing rhythm and amplitude. Since we were interested in the effects of breathing frequency, not amplitude, records whose amplitude were too different were discarded. ECG and breathing signals were sampled at $500 \mathrm{~Hz}$ in eight subjects and at $4 \mathrm{kHz}$ in twelve subjects by a plugin PC data-acquisition card (DT2821, Data Translation) and stored for further processing. RR time series were derived in two steps. The first step yielded an estimated location of each QRS complex by detecting when the ECG signal, low-pass filtered at $30 \mathrm{~Hz}$ and further differentiated, exceeded a threshold set at $75 \%$ of the maximal amplitude of the filtered signal. The second step corrected the estimated location by maximizing the correlation between the first QRS detected and the following ones. After properly detecting each QRS, the tachogram was computed by differentiating the position of each QRS. From the tachogram, a resampled RR time series was obtained with a sampling frequency of $4 \mathrm{~Hz}$ by interpolating with cubic splines. In order to remove low-frequency components present in the tachogram but unrelated to breathing patterns, the tachogram was high-pass filtered with a Butterworth fourth-order bidirectional filter with cut-off frequency at $0.05 \mathrm{~Hz}$. Next, the periodogram was estimated for each subject and each stage, and the $\mathrm{LF} / \mathrm{HF}_{\mathrm{n}}$ was computed.

Figure 3 summarizes the results for the 20 subjects. Both sampling frequencies yielded the same results. The $\mathrm{LF} / \mathrm{HF}_{\mathrm{n}}$ index is largest when breathing freely and smallest when

Table 2. Significance of the differences between $L F / H F_{n}$ for the different breathing stages. If $p<0.05$, the difference is considered to be significant.

\begin{tabular}{llllr}
\hline & Periodic 1 & FM & Periodic 2 & Free 2 \\
\hline Free 1 & $\mathbf{0 . 0 0 0 9}$ & $\mathbf{0 . 0 2 5 4}$ & $\mathbf{0 . 0 0 0 6}$ & 0.1926 \\
Periodic 1 & & $\mathbf{0 . 0 0 1 4}$ & 0.3390 & $<\mathbf{0 . 0 0 0 1}$ \\
FM & & & $\mathbf{0 . 0 1 4 4}$ & $\mathbf{0 . 0 0 0 3}$ \\
Periodic 2 & & & & $<\mathbf{0 . 0 0 0 1}$ \\
\hline
\end{tabular}




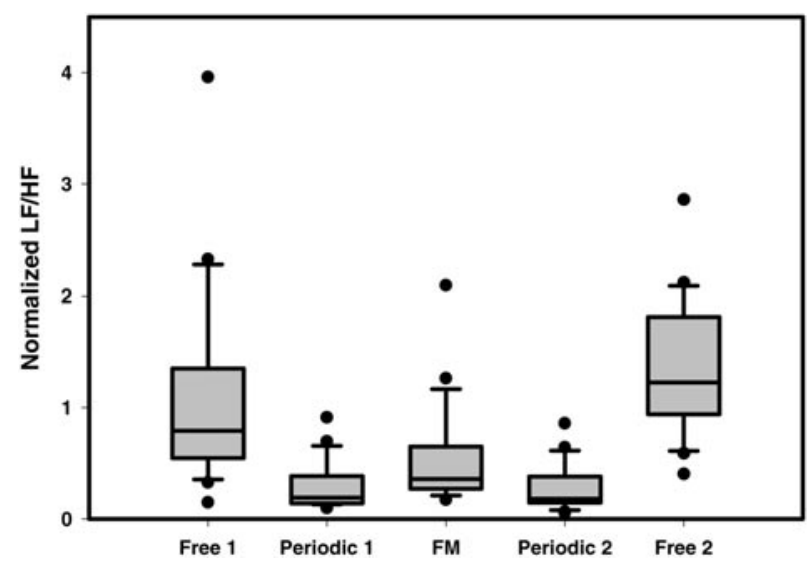

Figure 3. $\mathrm{LF} / \mathrm{HF}_{\mathrm{n}}$ index for the 20 subjects measured and the five stages (starting and finishing with breathing at will- 'free'). Mean, standard deviation, fifth and 95th percentiles, and maximum and minimum are represented for each stage.

breathing periodically. FM breathing yields an intermediate index value. Differences between stages were tested by a paired Student $t$-test whose results are shown in table 2 . There are no significant differences between the two stages of free breathing, nor between the two stages of periodic breathing. There are significant differences between free and periodic breathing between free and FM breathing and between periodic and FM breathing.

\section{Discussion}

The experimental results for periodic breathing show an $\mathrm{LF} / \mathrm{HF}_{\mathrm{n}}$ index smaller than that for FM breathing, the same as for the simulated data in figure $1\left(\right.$ signal $\left.R_{p}\right)$. Nevertheless, the $\mathrm{LF} / \mathrm{HF}_{\mathrm{n}}$ index is larger for experimental than for simulated data. This is because the LF band of experimental data includes other modulations, such as those of the baroreceptor that are not completely rejected by the high-pass filter applied to the tachogram. It may also be due to drifts of the mean heart rhythm, to the non-sinusoidal waveform of the respiratory sinus arrhythmia and to changes in the transfer function between the breathing rhythm and the heart rate.

FM breathing yields a large $\mathrm{LF} / \mathrm{HF}_{\mathrm{n}}$ index as predicted from the theoretical analysis and from simulated data $\left(R_{\mathrm{FM}}\right)$. Free breathing yields even larger values for the $L F / H F_{n}$ index than FM breathing, which is consistent with the large variability in breathing patterns when subjects are allowed to breathe at will. Therefore, interpreting changes in the $\mathrm{LF} / \mathrm{HF}_{\mathrm{n}}$ index requires us to consider the breathing patterns simultaneous with the recorded RR series.

\section{Conclusions}

The respiratory sinus arrhythmia reflects the relationship between the autonomic nervous system and the cardiovascular system, but it also reflects the breathing pattern. Any variation in this pattern influences the RR time series and modifies its power spectrum. Periodic breathing yields low values for the $\mathrm{LF} / \mathrm{HF}_{\mathrm{n}}$ index. Breathing at will yields the larger $\mathrm{LF} / \mathrm{HF}_{\mathrm{n}}$ values. Therefore, comparisons between the $\mathrm{LF} / \mathrm{HF}_{\mathrm{n}}$ index for different subjects in HRV studies must consider the actual breathing pattern. 


\section{References}

Arai Y, Saul J P, Albrech P et al 1989 Modulation of cardiac autonomic activity during and immediately after exercise Am. J. Physiol. $256 \mathrm{H} 132-\mathrm{H} 141$

Bigger J T, Fleiss J L, Rolnitzky L M, Steinman R C and Schneider W J 1991 Time course of recovery of heart period variability after myocardial infarction J. Am. Coll. Cardiol. 18 1643-9

Kleiger R E, Bosner M S, Rottman J N and Stein P K 1993 Time-domain measurements of heart rate variability J. Ambul. Monit. 6 1-18

Kollai M and Mizsei G 1990 Respiratory sinus arrhythmia is a limited measure of cardiac parasympathetic control in man J. Physiol. 424 329-42

Mateo J and Laguna P 2000 Improved heart rate variability signal analysis from the beat occurrence times according to the IPFM model IEEE Trans. Biomed. Eng. 47

Pagani M et al 1986 Power spectral analysis of heart rate and arterial pressure variabilities as a marker of sympathovagal interaction in man and conscious dog Circ. Res. 59 178-93

Pagani M, Malfatto G, Pierini S et al 1988 Spectral analysis of heart rate variability in the assessment of autonomic diabetic neuropathy Automedica 4 155-67

Peebles P Z 1976 Communication System Principles (Reading, MA: Addison-Wesley)

Pomeranz B et al 1985 Assessment of autonomic function in humans by heart rate spectral analysis Am. J. Physiol. 248 H151-H153

Sands K E, Appel M L, Lilly L S, Schoen F J, Mudge G H and Cohen R J 1989 Power spectrum analysis of heart rate variability in human cardiac transplant recipients Circulation 79 76-82

Task Force of the European Society of Cardiology and the North American Society of Pacing and Electrophysiology 1996 Heart rate variability, standards of measurement, physiological interpretation, and clinical use Eur. Heart J. 17 354-81

Wada T, Mizuno H, Ohba F and Kawashima H 1993 Nonlinear analysis of heart rate variability using bispectrum Annu. Int. Conf. IEEE Eng. Med. Biol. Soc. vol 15, pp 918-19

Womack B F 1971 The analysis of respiratory sinus arrhythmia using spectral analysis and digital filtering IEEE Trans. Biomed. Eng. 18 399-409 